\title{
Bevölkerungsrückgang als Problemgenerator für alternde Gesellschaften
}

Der „demografische Wandel“ ist ins Zentrum der öffentlichen Auseinandersetzungen gerückt. Anders als die Enquête-Kommission des Deutschen Bundestags (1990-2002) sieht der vorliegende Beitrag die Ursache der sozialpolitischen Probleme nicht in der zunehmenden Langlebigkeit, sondern in der Nachwuchsschwäche der deutschen Gesellschaft. Deren Folgen für Wirtschaftswachstum, Verteilungskonflikte und die Situation der älteren Generationen werden skizziert.

\section{Aussagekraft und Grenzen der Demografie}

Demografische Sachverhalte gehören zu den übersichtlichsten der Sozialwissenschaften. Sie sind ziemlich eindeutig und weitgehend verlässlich, denn ihre Erhebung beruht auf gesellschaftsweiten administrativen Routinen. Die Zahl der zu berücksichtigenden Variablen ist sehr beschränkt: Im Zentrum stehen Alter, Geschlecht, Geburten, Wanderungen und Todesfälle, daneben spielen räumliche Abgrenzungen und die Zivilstandsdaten sowie die Nationalität eine strukturierende Rolle. Was eine Bevölkerung ist, bestimmt sich nach behördlichen Regeln.

Die Demografie hat früh den Anschluss an die mathematische Statistik gefunden. Bevölkerungsmodelle gehören zu den ältesten sozialwissenschaftlichen Modellen. Ihre Fortentwicklung erlaubt heute Bevölkerungsvorausschätzungen von einer in den übrigen Sozialwissenschaften unbekannten Verlässlichkeit. Wenn sie als Prognosen missverstanden und daher durch die spätere Wirklichkeit dementiert werden, so liegt es in der Regel an falschen Annahmen, die selbst nicht mehr demografischer Natur sind. Beispiele für solche Fehlerquellen sind die Wirtschaftsentwicklung, das Auftreten von Kriegen oder Epidemien, das Aufkommen neuer Methoden der Geburtenkontrolle oder Wandlungen im Bereich der Geschlechter- und Familienverhältnisse.

Allerdings bleibt eine Demografie, die sich nur im Rahmen ihrer vertrauten Variablen bewegt, interdisziplinär wenig anschlussfähig. Die heute offenen und deshalb spannenden Fragestellungen beziehen sich auf Ursachen und Folgen der Bevölke- rungsentwicklung oder - wie man in der Bundesrepublik heute sagt - des „Demografischen Wandels“. Mit dieser Formulierung wird das Thema schon auf aktuelle Probleme eingeengt, insbesondere auf die zunehmende Langlebigkeit und die sinkenden Geburtenzahlen, aber auch auf Veränderungen in den Wanderungsströmen. Immer neue und verfeinerte Beschreibungen dieser Phänomene helfen uns praktisch-politisch allerdings nicht viel weiter, solange es an Wissen über ihre Folgen und die Bedingungen ihrer Beeinflussbarkeit fehlt. Die Demografie ist deshalb heute herausgefordert, sich zu einer interdisziplinär orientierten „Bevölkerungswissenschaft" (Population Studies) weiter zu entwickeln. Leider ist die universitäre Präsenz der Demografie, und erst recht der Bevölkerungswissenschaft, in Deutschland minimal und in jüngster Zeit sogar noch reduziert worden. Das ist fatal für ein Land, dessen demografische Perspektiven zu den ungünstigsten der Welt gehören.

Die im Vergleich zum empirischen Wissen der übrigen Sozialwissenschaften hohe Verlässlichkeit demografischen Wissens resultiert nicht aus den sozialen, sondern aus den biotischen Komponenten seiner Grundlagen. Die Zähleinheiten der Demografie sind natürliche Personen, Menschen mit einer beschränkten, aber unter modernisierten Bedingungen im Vergleich zu früher längeren und weniger ungleichen Lebensspanne. Der ungebrochene Trend einer Verlängerung der mittleren Lebenserwartung seit der Geburt hat in Westeuropa um die Mitte des 18. Jahrhunderts begonnen, zunächst vor allem durch die Verminderung der Säuglings- und Kindersterblichkeit infolge verbesserter Hygiene und Ernährungsbedingungen. In dem Maße, wie im 20. Jahrhundert die Todesursachen im Kindes- und jüngeren Erwachsenenalter durch Fortschritte von Prävention und
Therapie eliminiert worden sind, gewann der Rückgang der Alterssterblichkeit an statistischem Gewicht: So hat in der früheren Bundesrepublik zwischen 1972 und 1999 die mittlere Lebenserwartung bei der Geburt für beide Geschlechter um $10 \%$, für die 65-Jährigen um $26 \%$ und für die 80-Jährigen um $33 \%$ zugenommen. Wie insbesondere die Studien am Rostocker Max-Planck-Institut (MPI) für demografische Forschung (Oeppen/Vaupel 2002) nahelegen, ist weiterhin mit einem verstärkten Rückgang der Alterssterblichkeit zu rechnen, sodass in dieser Hinsicht die Bevölkerungsvorausberechnungen des Statistischen Bundesamtes eher konservativ erscheinen.

\section{Demografisches Altern ist wünschenswert}

Während der Sterblichkeitsrückgang bei gleichbleibender Fertilität im 18. und 19. Jahrhundert im Wesentlichen zu einer Verjüngung der Bevölkerung beitrug, wird er im 21. Jahrhundert nachhaltig zur Alterung der Bevölkerung beitragen. Infolge dessen entsteht das biologisch, sozial und kulturell neue Phänomen einer massenhaften Verbreitung der jungen Alten, eine bisher gesellschaftlich unterbestimmte neue Lebensphase zwischen dem Ausscheiden aus dem

Franz-Xaver Kaufmann, Professor em. für Sozialpolitik und Soziologie an der Universität Bielefeld. Mitglied der NordrheinWestfälischen Akademie der Wissenschaften; zurzeit Berater des "Forum Demographischer Wandel" des Bundespräsidenten. e-mail: f.x.kaufmann@uni-bielefeld.de 
Erwerbsleben und der Phase zunehmender Hinfälligkeit, mit der in der Vergangenheit das Alter meist in Verbindung gebracht wurde. Frank Schirrmachers irritierendes „Methusalem-Komplott" (Schirrmacher 2004) ist da offenkundig in eine auch von der Bevölkerung empfundene Lücke gestoßen.

Die Zunahme der Lebenserwartung vollzieht sich weitgehend unabhängig von der Entwicklung der Fertilität. Sie ist die Frucht der wirtschaftlichen, sozialen und medizinischen Fortschritte der letzten 250 Jahre. Und sie vollzieht sich vor dem kulturellen Hintergrund des Glaubens an den eigenständigen Wert, an die Würde eines jeden Menschenlebens, wie dies auch in Art. 1 unseres Grundgesetzes mit der wünschenswerten Deutlichkeit ausgesprochen wird.

Hätte sich dieser säkulare Sterblichkeitsrückgang bei unverminderter Fertilität vollzogen, so wäre es im 20. Jahrhundert zu einem explosiven Bevölkerungswachstum in Europa gekommen, wie in manchen Entwicklungsländern. Deshalb war die Beschränkung der Geburten, die sich zuerst in Frankreich ab etwa 1830 verbreitete, eine notwendige Anpassungsreaktion, die zwangsläufig zu einem Altern der Bevölkerung führte. Die Geburtenkontrolle erfasste im 20. Jahrhundert immer größere Teile der Bevölkerung in Europa. Deutschland war dabei eher ein „später Starter“, aber auch ein „schneller Sprinter", denn innerhalb einer Generation - zwischen den Geburtsjahrgängen 1865 und 1900 - sank die Kohortenfertilität, also die mittlere Kinderzahl eines Frauenjahrgangs (CFR/ Completed Fertility Rate), von nahezu fünf auf zwei Kinder (Abbildung 1, dicke Kurve, oberer und rechter Maßstab). Da der Anteil der verheirateten Frauen in diesem Zeitraum zugenommen hat, war der Rückgang der durchschnittlichen Kinderzahl pro Ehe noch ausgeprägter. Dass die Kurve der allgemeinen Geburtenziffer (Abbildung 1, dünne Kurve, linker und unterer Maßstab) zumeist über derjenigen der Kohortenfertilität bis ca. 1963 liegt, ist eine Folge des Sterblichkeitsrückgangs in den jüngeren Lebensjahren, wodurch die Anzahl an Frauen im gebärfähigen Alter zugenommen hat. Dieser Effekt verlor im Laufe des 20. Jahrhunderts zunehmend an Einfluss, und das umgekehrte Verhältnis der beiden Kurven nach 1963 ist im Wesentlichen auf die Erhöhung des mittleren Gebäralters zurückzuführen.

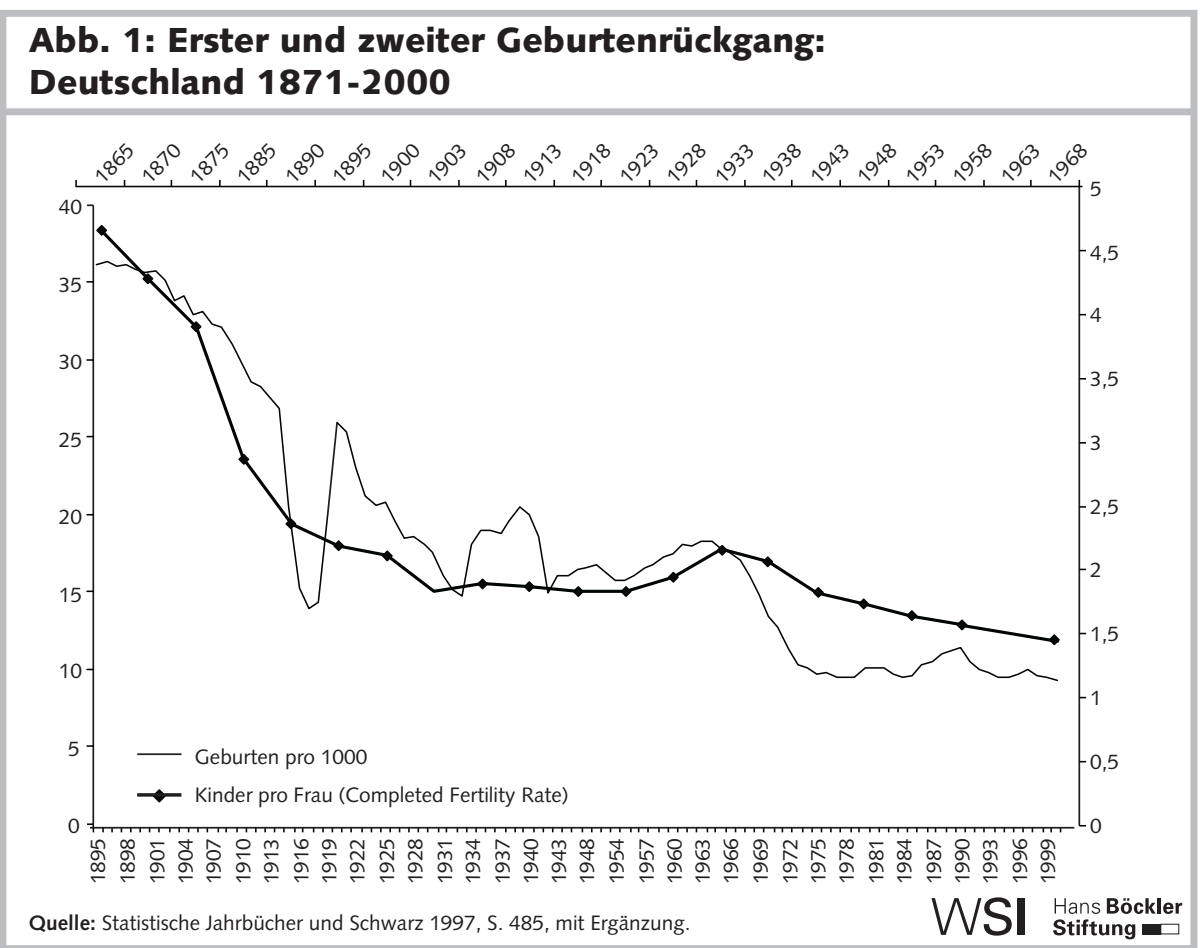

Die Demografen sprachen hinsichtlich des „ersten Geburtenrückgangs“ (hier zwischen 1900 und 1935) von „Demographic Transition". Sie formulierten die theoretische Annahme, dass dem zunächst einsetzenden Sterblichkeitsrückgang nach einigen Jahrzehnten zwangsläufig ein Geburtenrückgang folge. Dadurch führe die Bevölkerungsentwicklung von einem für traditionale Gesellschaften charakteristischen Gleichgewicht bei hoher Sterblichkeit und hoher Fruchtbarkeit zu einem neuen, für moderne Gesellschaften charakteristischen Gleichgewicht bei niedriger Sterblichkeit und niedriger Fruchtbarkeit. Charakteristisch für die Übergangsphase sei zunächst ein starkes Bevölkerungswachstum, dem im Zuge seiner Abschwächung eine Phase des demografischen Alterns folge. So lange das demografische Altern sich im Rahmen eines reproduktiven Gleichgewichts vollzieht, ist es in wirtschaftlicher und vermutlich auch sozialer Hinsicht weitgehend unproblematisch, ja sogar Zeichen einer erfolgreichen demografischen Modernisierung. Vielfach geäußerte Befürchtungen, die Zunahme des Anteils alter Menschen würde per se zu einer Verengung des politischen Zeithorizonts und zu politischer Unbeweglichkeit führen, lassen sich sozialwissenschaftlich nicht erhärten (Kaufmann 2007).

\section{Bevölkerungsrückgang infolge von Nachwuchs- schwäche}

Von einem demografischen Gleichgewicht in traditionalen Gesellschaften zu sprechen, ist ohnehin eine heroische Vereinfachung. Für moderne Gesellschaften zeigt die Geburtenentwicklung der letzten Jahrzehnte erst recht einen weiteren Geburtenrückgang, der das reproduktive Gleichgewicht verletzt. Der brüske „Zweite Geburtenrückgang“ (in Deutschland von 19651975) kam durch das Zusammentreffen der Entdeckung praktikablerer und sicherer Formen der Empfängnisverhütung mit einer emanzipativen Veränderung der weiblichen Lebensbedingungen durch Bildung, außerhäusliche Berufstätigkeit und Wertewandel zustande, bei fortdauernden paternalistischen Einstellungen auf Seiten der Männer. Dies hat im Falle Deutschlands dazu geführt, dass sich die Bevölkerung durch Geburten nur noch zu zwei Dritteln reproduziert, und diese Nachwuchsschwäche dauert nun schon eine Generation an. Das heißt konkret: Seit 1975 haben 1.000 Frauen nur noch 667 Töchter, die das Fortpflanzungsalter erreichen; von diesen Töchtern können sie unter gleichbleibenden Annahmen noch 445 Enkelinnen, und von diesen nur noch 297 Urenkelinnen erwarten. 


\section{Abb. 2: Bevölkerungsstand 1950 bis 2000 nach Vorausberechnungen bis 2050 bzw. 2100 (Deutschland)}

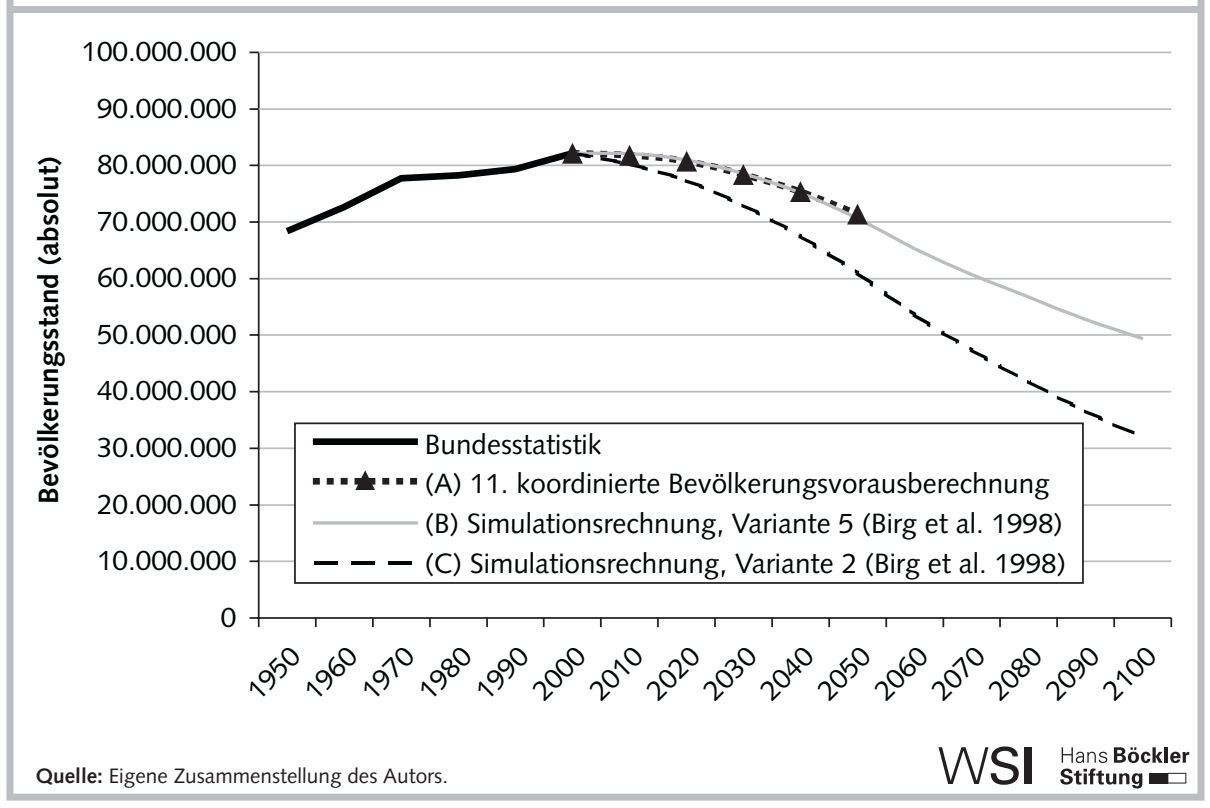

An dieser Stelle sei angemerkt, dass sich die Fertilität in Deutschland während des 20. Jahrhunderts stärker als in anderen Teilen Europas reduziert hat. Schon um 1950 hatte Deutschland eine niedrigere Fertilität als die wichtigsten Vergleichsländer. Ähnlich wie Frankreich im 19. Jahrhundert erscheint Deutschland heute als demografisches „Pionierland“, und zwar hinsichtlich der Lowest Fertility, wie Demografen das Absinken der Fertilität unter einen Wert von 1,5 bezeichnen. Ab dieser Größenordnung wird es aufgrund eines demografischen Verstärkereffektes zunehmend schwieriger, den regressiven Trend umzukehren. In dieselbe Richtung wirken auch theoretisch zu erwartende und in Deutschland bereits empirisch beobachtbare Einstellungsänderungen: Die Enttäuschung der in den letzten Jahrzehnten aufgebauten Hoffnung auf dauerhafte Wohlstandsmehrung verdüstert die Zukunftserwartungen. Und überdies scheint Kinderlosigkeit mit ihrer faktischen Verbreitung zunehmend auch normativ "salonfähig“ zu werden. Dorbritz (2005) spricht sogar von einer entstehenden „Kultur der Kinderlosigkeit“. Etwa ab dem Geburtsjahrgang 1950 polarisiert sich die Bevölkerung in Deutschland in Familien und Kinderlose.

Inzwischen ist Deutschland hinsichtlich der Fertilität von einigen Ländern Südeuropas und neuerdings auch Osteuropas „unterholt" worden. Wenn man allerdings berücksichtigt, dass Frauen deutscher Nationalität im Durchschnitt nur ca. 1,1 Kin- der zur Welt bringen, so zeigt sich, dass die Verhältnisse für die einheimische Bevölkerung hierzulande keineswegs günstiger sind als in Süd- und Osteuropa. Es gibt also keine „natürliche“ Untergrenze des Geburtenrückgangs!

Der eigentliche Problemgenerator im Kontext der demografischen Entwicklung ist nicht der Sterblichkeitsrückgang, sondern die niedrige Fertilität. Ihr demografisches Gewicht wird im deutschen Falle durch die zwar stark schwankenden, aber im Durchschnitt der letzten Jahrzehnte hohen Zuwanderungssalden von ca. 170.000 Menschen pro Jahr verschleiert.

Abbildung 2 zeigt die Veränderung der Bevölkerungsgröße in Deutschland zwischen 1950 und 2050 unter der wahrscheinlichsten Annahme des Statistischen Bundesamtes von 2006, ferner eine mit ähnlichen Annahmen operierende Vorausberechnung durch Herwig Birg für das ganze 21. Jahrhundert (Birg et al. 1998). ${ }^{1}$ Die Annahmen, die der Darstellung zugrunde liegen, machen deutlich, wie sehr ein kontinuierlicher Zuwanderungssaldo von 150.000 Personen den Bevölkerungsrückgang abbremst: Ohne Zuwanderung würde sich der Bevölkerungsstand bis zum Jahre 2080 gegenüber dem Höchststand um 2010 halbieren. Schon seit 1972 reichen die Geburten nicht mehr aus, um die Sterbefälle zu kompensieren, und ab ca. 2010 wird sich auch der kompensierende Effekt der Zuwanderung im bisherigen Umfang erschöpfen und die Bevölkerung aller Vor- aussicht nach zunächst geringfügig, aber allmählich immer stärker zurückgehen. ${ }^{2}$ Demografische Wachstums- und Schrumpfungsprozesse entfalten unter gleichbleibenden Annahmen eine zunehmende Wucht: Je stärker die Entwicklung vom Bevölkerungsgleichgewicht abweicht und je länger die Schrumpfung andauert, desto schwieriger wird es, die Tendenz zu verändern. Das ist im Wesentlichen auf den überproportionalen Rückgang der Frauen im gebärfähigen Alter zurückzuführen.

Anhand mehrerer Modelle von Birg et al. (1998) lässt sich der differenzielle Einfluss von Fertilität, Mortalität und Wanderungen auf den Bevölkerungsrückgang im 21. Jahrhundert abschätzen. Der Sterblichkeitsrückgang zeitigt nur bescheidene Wirkungen auf die Schrumpfungsrate. Für das Verhältnis von Geburten und Zuwanderungssaldo kann man davon ausgehen, dass eine Veränderung der langfristigen Fruchtbarkeitsrate von 0,2 in etwa einer Veränderung des langfristigen jährlichen Zuwanderungssaldos von 120.000 Personen entspricht. Selbst bei einer Erhöhung der Fertilität auf 1,6 Kinder würde ein $\mathrm{Zu-}$ wanderungssaldo von jährlich 300.000 Personen nur knapp zur Erhaltung des Bevölkerungstandes ausreichen. Da nach bisherigen Erfahrungen sich nur jeder dritte bis vierte Zuwanderer dauerhaft in der Bundesrepublik niederlässt, bedürfte es selbst unter diesen optimistischen Fertilitätsannahmen einer fortgesetzten jährlichen Zuwanderung von rund einer Million Personen, um den Bevölkerungsstand zu halten - ein sehr unwahrscheinliches und zudem konfliktträchtiges Szenario. Ein sich beschleunigender Bevölkerungsrückgang ist somit für das 21. Jahrhundert in Deutschland mit hoher Wahrscheinlichkeit zu erwarten, lediglich dessen Ausmaß steht einer politischen Beeinflussung offen.

1 Vgl. Statistisches Bundesamt (2006, Anhang B). Angegeben ist der Mittelwert zwischen den Varianten W 1 und W 2 der "mittleren Bevölkerung "; d.h., es wird mit einem jährlichen Zuwanderungssaldo von 150.000 Personen gerechnet, bei einer konstanten Fertilität von 1,4 Kindern pro Frau und mäßigem weiterem Sterblichkeitsrückgang. Der Variante 5 von Birg et al. (1998) liegen sehr ähnliche Annahmen zugrunde. In Variante 2 von Birg et al. (ebd.) wird dagegen mit einem Wanderungssaldo von 0 gerechnet, d.h., die Bevölkerungsentwicklung resultiert ausschließlich aus dem jährlichen Saldo der Geburten und Todesfälle.

2 Da der Zuwanderungssaldo in jüngster Zeit stark zurückgegangen ist, hat der Bevölkerungsrückgang inzwischen bereits begonnen. 
Einigermaßen gut dokumentierte Fälle eines historischen Bevölkerungsrückgangs betreffen das römische Reich in seiner Spätphase sowie Spanien im 17. und 18. Jahrhundert. In neuerer Zeit kommt Frankreich unserer Problemstellung am nächsten. Hier stagnierte die Bevölkerung zwischen 1850 und 1950 weitgehend, war jedoch landesweit noch nicht rückläufig. Besser dokumentiert sind rückläufige regionale Entwicklungen, darunter auch hoch industrialisierter Stadtgebiete, die mit dem technischen Wandel einer zunehmenden Deindustrialisierung anheimfielen. In allen bekannten Fällen ging Bevölkerungsstagnation oder gar Rückgang mit politischem Bedeutungsverlust, ökonomischer Stagnation und fehlender Bereitschaft zu innovativem Wandel einher. So hält Sauvy (1968, S. 355) fest: „Not one single historical or present-day instance can be cited of a declining or stagnating population that has enjoyed any real economic expansion."

Allerdings bleiben bei derartigen korrelativen Behauptungen die Kausalitäten unklar. Es ist ja zunächst plausibel, dass günstige ökonomische Umstände Zuwanderung und ungünstige ökonomische Umstände Abwanderung zur Folge haben, wie wir dies derzeit auch in Ostdeutschland beobachten können. Auch die politische Bedeutung und die soziale Wohlfahrt scheinen zum Mindesten heute stärker mit dem ökonomischen als mit dem demografischen Potenzial verbunden. Inwieweit kann die demografische Entwicklung dennoch als unabhängige Variable, also als primärer Wirkfaktor betrachtet werden? Der „zweite Geburtenrückgang" seit der Entdeckung und Verbreitung der Ovulationshemmer und anderer, vom Geschlechtsverkehr unabhängiger Formen der Empfängnisverhütung ist ja eine endogene Veränderung, die nichts mit ökonomischen oder politischen Krisen zu tun hat. Er ist vielmehr Ausdruck einer gerade durch Wohlstand gewonnenen Freiheit, insbesondere der Frauen, die sich heute auch unabhängig vom Joch oder den Freuden der Ehe zu behaupten vermögen.

Entsprechende Argumente lassen sich nur durch analytische Isolierung des demografischen Faktors gewinnen. In der Wirklichkeit müssen wir aber stets von einer engen Interdependenz zwischen ökonomischen, politischen und demografischen Faktoren ausgehen. Das gilt umso mehr, als demografische Veränderungen in der Regel sehr langsam ablaufen.

\section{Wirtschaftliche Folgen eines Bevölkerungs- rückgangs}

Ein zentrales Argument haben bereits John Maynard Keynes (1937) und Alwin Hansen (1939) formuliert: Sie führten die Intensität der Weltwirtschaftskrise auf den Wegfall des Bevölkerungswachstums zurück, das im 19. Jahrhundert und bis zum Ersten Weltkrieg die starke wirtschaftliche Expansion mitgetragen hatte. In seiner „Theorie der säkularen Stagnation“ argumentierte Hansen, das bisherige Wirtschaftswachstum der Industrieländer habe vor allem auf drei Investitionsanreizen beruht, nämlich der Entdeckung neuer Territorien und Ressourcen, dem Bevölkerungswachstum sowie innovativen Erfindungen. Die beiden erstgenannten ,extensiven “ Investitionsgelegenheiten verlören an Bedeutung, sodass zukünftige Wachstumsimpulse nur noch von Erfindungen und technischen Neuerungen zu erwarten seien. In einer Volkswirtschaft ohne Bevölkerungszuwachs sei deshalb Vollbeschäftigung nur durch eine erhöhte Innovationsrate im Bereich der wirtschaftlichen Güter und damit verbundener Verbreiterung der Wohlfahrt in der gesamten Bevölkerung zu erreichen. Die technischen Fortschritte und die Ausbreitung wohlfahrtsstaatlicher Einrichtungen und Umverteilungsprozesse nach dem Zweiten Weltkrieg schienen zunächst als Antwort auf die Herausforderung zu genügen, und zudem rückte nun die Bevölkerungszunahme in der Dritten Welt ins Zentrum der Bevölkerungsfrage.

Keynes und Hansen haben den Konsumaspekt und damit den drohenden Nachfrageausfall infolge eines Bevölkerungsrückgangs betont. Wir müssen heute die Argumentation um den rascher wirksamen Produktionsaspekt ergänzen. Dieser lässt sich am besten anhand humankapitaltheoretischer Überlegungen verdeutlichen, welche in der orthodoxen deutschen Wirtschaftstheorie allerdings noch wenig aufgenommen werden. ${ }^{3}$ Im Zuge des Übergangs

3 Wegweisend für die Verbindung von Humankapital- und Wachstumstheorie ist Romer (1990); vgl. auch Kaufmann (2005, S. 86ff.). Berkel et al. (2004) haben diesen Ansatz in einem empirisch unterlegten Simulationsmodell umgesetzt. 


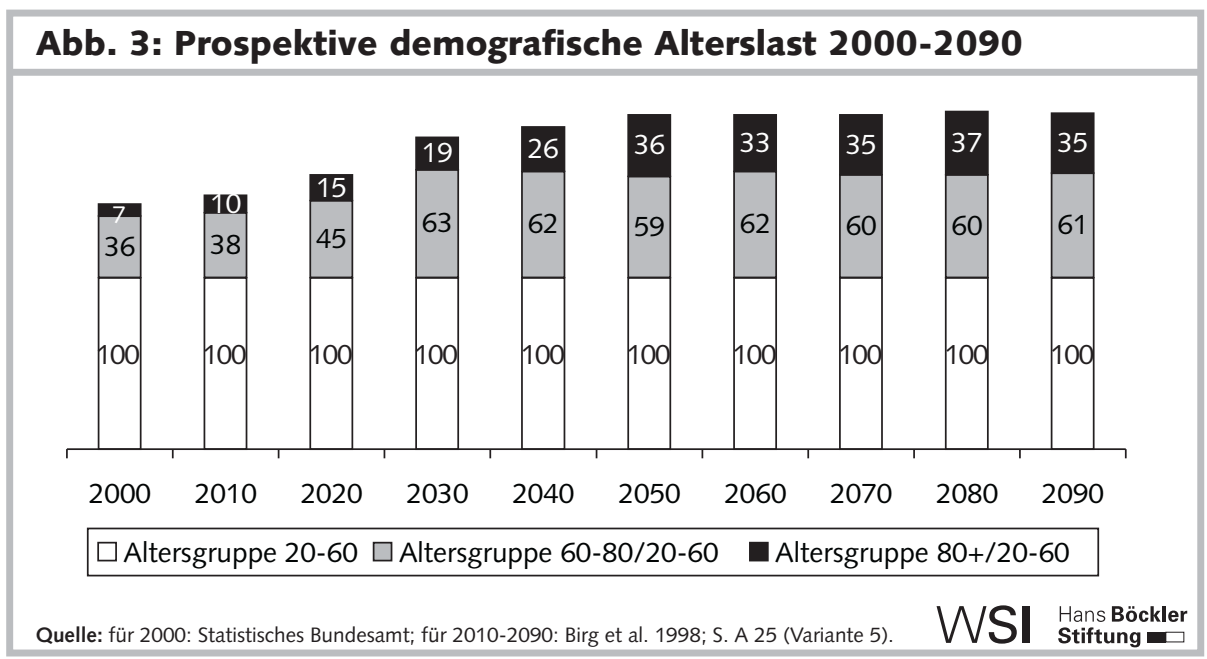

zur Dienstleistungsökonomie und zur „Wissensgesellschaft“ ist die Zunahme der Arbeitsproduktivität immer weniger vom Wachstum des Sachkapitals und immer stärker von der Zunahme des Humankapitals abhängig. Soweit es nicht gelingt, den vorhandenen Nachwuchs entsprechend besser zu qualifizieren, hat der Geburtenrückgang eine Schwächung des beruflichen Nachwuchses zur Folge, der in der Regel auch ein wichtiger Faktor für die Verbreitung technischer Innovationen ist. Allerdings verdeutlicht die Humankapitaltheorie auch, dass es nicht einfach auf die demografische Entwicklung, also auf die $\mathrm{Zu}$ oder Abnahme der Köpfe ankommt, sondern auf das, was in ihnen steckt. In diesem Sinne kommen Berkel et al. (2004, S. 86) zu dem Ergebnis: „Eine langfristige Stärkung des Bruttonationaleinkommens pro Kopf entsteht durch eine höhere Geburtenrate nur dann, wenn die zusätzlich geborenen Kinder auch besser ausgebildet werden. Entscheidend für ein langfristiges Wachstum ist daher das zukünftige Humankapital und nicht per se eine höhere Geburtenrate."

Im deutschen Falle ist zu vermuten, dass infolge der geringen Durchschnittsqualifikation der Zuwanderer der relative Verlust an Humankapital noch stärker ausfällt als der Rückgang der nachwachsenden Generationen. Zudem zeigen empirische Untersuchungen ein Stagnieren der Bildungsaufwendungen für die nachwachsende Generation (Ewerhart 2003; Henke 2005). Eine Kompensation des Geburtenrückgangs durch verstärkte Qualifikation des vorhandenen Nachwuchses hat in den letzten Jahrzehnten nicht stattgefunden.

Obwohl grundsätzlich der Wegfall des Bevölkerungswachstums bei gleichblei- bender Produktivitätssteigerung eine stärkere Steigerung der Pro-Kopf-Einkommen und damit einen Wohlfahrtsgewinn ermöglichen würde, sprechen dieser und weitere Gründe dafür, dass in einer stagnierenden oder gar rückläufigen Erwerbsbevölkerung die Rate der Produktivitätssteigerung ebenfalls sinkt. Nimmt man die Wirkungen der Globalisierung hinzu, die im Rahmen der internationalen Arbeitsteilung den Ländern mit hohem Wohlstand nur noch innovative und hoch produktive Tätigkeiten zugesteht, so wird deutlich, dass es Deutschland nur mit ganz außergewöhnlichen Bildungsanstrengungen und besonderer Förderung der bildungsfernen Schichten gelingen könnte, den Nachwuchsmangel in etwa auszugleichen.

Der erste Pfad, auf dem sich der Bevölkerungsrückgang als Problemgenerator für alternde Gesellschaften erweist, bezieht sich somit auf das trendmäßig geringere, im ungünstigen Fall sogar negative Wirtschaftswachstum. Es ist damit zu rechnen, dass der Trend sich bei gegebener Produktivität nicht nur proportional zum Rückgang der Gesamtbevölkerung, sondern proportional zum Rückgang der erwerbstätigen Bevölkerung reduziert. ${ }^{4}$ Angesichts der zu erwartenden Veränderungen der nachgefragten Qualifikationen - Rückgang bei den Lehrberufen, Zunahme bei den Hochschulabsolventen - dürfte die zu erwartende Humankapitallücke noch breiter ausfallen als die demografische Lücke (Meyer/Wolter 2007).

Darüber hinaus ist zu vermuten, dass Länder mit stark rückläufiger und alternder Erwerbsbevölkerung auch in geringerem Maße innovativ sind und daher geringere Raten der Produktivitätssteigerung erzielen als Länder mit einer günstigeren demografischen Konstellation. Von besonderer Bedeutung ist in diesem Zusammenhang der rückläufige Anteil der jährlich neu ins Erwerbsleben Tretenden im Verhältnis zur gesamten Erwerbsbevölkerung, denn die „Newcomer" lassen sich am leichtesten auf neue Technologien hin orientieren. Immerhin ließe sich dieser Anteil durch vermehrte Qualifikationsanstrengungen auch bei rückläufigen Jahrgangsstärken in etwa stabilisieren.

\section{Verteilungspolitische Folgen}

Neben die Frage nach dem Wachstum tritt die Frage nach der Verteilung des Sozialpro$d u k t s$. Selbst wenn es gelänge, den überproportionalen Rückgang der Erwerbsbevölkerung durch entsprechende Produktivitätssteigerungen zu kompensieren, wie die meisten ökonomischen Szenarien im Auftrag der jeweiligen Bundesregierung bisher suggeriert haben, könnte das Wohlstandsniveau der Rentner nur durch eine wesentliche Steigerung der Umverteilungsquote beibehalten werden.

Denn die erwerbstätige Bevölkerung wird mit dem allmählichen Ausscheiden der „Baby-Boomer“ bis 2035 rasant abnehmen, während die Population der 60- bis 80-Jährigen bis 2030 und diejenige der über 80-Jährigen sogar bis 2050 zunehmen wird. Dementsprechend steigt die demografische Alterslast zwischen 2010 und 2050 rapide an, um in der Folge auf der dann erreichten Höhe in etwa zu verharren (Abbildung 3). ${ }^{5}$ Selbst wenn es gelingen sollte, das mittlere Verrentungsalter um zwei Jahre zu erhöhen, was angesichts der Widerstände der Wirtschaft gegen die Einstellung älterer Arbeitskräfte bereits ein ehrgeiziges Ziel ist, wird sich eine stärkere Umverteilung zugunsten der älteren Generationen oder aber eine weitere Reduktion

4 Nach der jüngsten Bevölkerungsvorausberechnung des Statistischen Bundesamtes (2006) dürfte sich der Bestand an Personen zwischen 20 und 65 Jahren zwischen 2010 und 2050 um 22-29 \% reduzieren; für die Differenz entscheidend ist das Ausmaß der Zuwanderung.

5 Der Abbildung 3 liegt die in FN 1 erwähnte Annahme der Variante 5 von Birg et al. (1998) zugrunde. 
ihrer durchschnittlichen Renteneinkommen nicht vermeiden lassen.

Die hier zugrunde liegende Problematik lässt sich durch eine bevölkerungsanalytische Überlegung verdeutlichen. In einer geschlossenen Volkswirtschaft gilt unabhängig vom Modus der Finanzierung die sogenannte Mackenroth'sche Regel, der zufolge die Noch-nicht und die Nicht-mehr erwerbstätigen Generationen aus dem von den Erwerbstätigen und den Unternehmungen produzierten Sozialprodukt der gleichen Periode unterhalten werden müssen. Man kann deshalb fragen, welches Verhältnis von noch nicht erwerbstätigen Kindern und Jugendlichen einerseits und nicht mehr erwerbstätigen Senioren andererseits $\mathrm{zu}$ einer langfristigen Minimierung der Versorgungslasten für die erwerbstätige Bevölkerung führt. Dabei werden die Annahmen hinsichtlich der Erwerbsbeteiligung und der Bedürfnisse konstant gesetzt, das heißt, wir betrachten zunächst nur die demografischen Zusammenhänge.

Es lässt sich zeigen, dass unter den gegenwärtig herrschenden Sterblichkeitsverhältnissen das langfristige Minimum der Versorgungslasten erreicht wird, wenn die Geburtenhäufigkeit gerade die jeweiligen Generationen ersetzt, also bei etwa 2,1 Kindern pro Frau. ${ }^{6}$ Aber es gilt auch: Je geringer die Sterblichkeit, desto höher ist die versorgungsoptimale Fertilität. Anschaulich ausgedrückt: Je mehr Alte zu versorgen sind, desto mehr Nachwuchs ist erforderlich. Noch brisanter wird die Analyse, wenn wir die Annahmen bezüglich der zu befriedigenden Bedürfnisse variieren. Nimmt man im Anschluss an empirische Schätzungen an, dass die Versorgung der nachwachsenden Generation pro Kopf nur zwei Drittel derjenigen der alten Generation kostet, so läge das langfristige Minimum der Versorgungslasten bei einer Reproduktion von $130 \%$, und dementsprechend stellt sich die gegenwärtige Reproduktion von $65 \%$ noch ungünstiger dar.

Die hier modellartig vorgeführten volkswirtschaftlichen Zusammenhänge lassen sich zu der These verdichten, dass eine niedrige Fertilität umso unschädlicher ist, je geringer die Pro-Kopf-Aufwendungen für die alte Generation im Verhältnis zu denjenigen der nachwachsenden Generation sind. So gilt unter gegenwärtigen $\mathrm{Be}-$ dingungen eindeutig: Je geringer der Nachwuchs, desto schwieriger ist langfristig der Unterhalt der alten Generation. Gelänge es, durch massive Verstärkung von Familien- und Bildungspolitik die Humankapitalbildung zu erhöhen, auch auf Kosten der aktuellen Aufwendungen für die alte Generation, so würden sich auf Dauer auch die alten Generationen besser stellen.

Die bisherigen Überlegungen standen unter der Prämisse einer geschlossenen Volkswirtschaft und haben den Finanzierungsmodus nicht berücksichtigt. Heute ist es so, dass der Unterhalt der älteren Generation nahezu vollständig über staatlich organisierte Umverteilung finanziert wird, der Unterhalt der nachwachsenden Generationen dagegen nur etwa zu einem Viertel. Die öffentlichen Haushalte werden also durch die demografische Entwicklung noch weit stärker gebeutelt. Für den von Ökonomen empfohlenen Ausweg einer stärkeren Kapitalfundierung der Renten mittels Kapitalexport ist das demografische "Gelegenheitsfenster" bereits weitgehend vorbei. Dennoch bleibt es weiterhin sinnvoll, die Altersvorsorge durch kapitalbildendes Sparen zu fördern, wie dies seitens der Bundesregierung mittels der sogenannten Riester-Rente angestrebt wird. Aber deren Freiwilligkeit wird die „Transferausbeutung der Familien“ (Borchert 1993) durch die Kinderlosen nicht mildern, sondern verstärken, denn die Kinderlosen können die hierfür notwendigen Ersparnisse wesentlich leichter aufbringen, und werden dafür auch noch subventioniert!

Eine massive und nachhaltige Umverteilung öffentlicher Mittel zugunsten der nachwachsenden Generationen ist aufgrund dieser Zusammenhänge unabweisbar. Dabei geht es nicht nur um eine "bevölkerungsbewusste Familienpolitik“ (Wingen 2003), sondern ebenso sehr um eine Stärkung der Bildungspolitik, insbesondere auch um eine die Sozialisationsbedingungen lernschwacher Jugendlicher berücksichtigende Vernetzung von Bildungspolitik und Jugendpolitik. Darüber hinaus gehören die Steuerpolitik und die Rentenpolitik auf den Prüfstand der Transfergerechtigkeit im Verhältnis von Eltern und Kinderlosen. Es wäre angemessen, die erforderlichen Umverteilungen zugunsten von Eltern und Kindern in den öffentlichen Haushalten hauptsächlich zulasten derjenigen vorzunehmen, die keine Aufwendungen für die Kindererziehung erbracht haben. Das deutsche Transfersystem begünstigt nach wie vor die Kinderlosen in ungebührlicher Weise und ist damit selbst eine Ursache für die es bedrohenden de- mografischen Entwicklungen (Borchert 2003). Altersvorsorge durch Sparen sollte für Kinderlose verpflichtend gemacht und ein Teil ihrer Ansprüche aus der Rentenversicherung zugunsten der Eltern von mehr als zwei Kindern umverteilt werden (Sinn 2003, S. 389ff.; Kaufmann 2005, S. 224ff.). Ein Abbau der Prämien für Kinderlosigkeit ist auch politisch unabweisbar, denn keine Regierung wird die Leistungen zugunsten von Eltern so sehr ausdehnen können, dass auf diese Weise die ökonomischen Vorteile der Kinderlosigkeit allein im Sinne eines Familienleistungsausgleichs reduziert werden könnten. ${ }^{7}$

\section{$P$ \\ Probleme der älteren Generationen}

Die anhaltend niedrige Fertilität verschärft die Problemlagen für Teile der älteren Generation nicht nur in ökonomischer, sondern auch in sozialer Hinsicht. Das gilt in besonderem Maße angesichts des deutschen Fertilitätsmusters, das durch eine zunehmende Polarisierung zwischen Familien und Kinderlosen gekennzeichnet ist. Während beispielsweise in Italien der Geburtenrückgang vorzugsweise durch eine Zunahme der Einkindfamilien erklärt werden kann, verzichten in der Regel junge Menschen in Deutschland entweder ganz auf Kinder oder wollen auch ein oder gar zwei Geschwister für ihr erstes Kind. Diese Polarisierung ist nicht nur ein statistisches sondern auch ein sozialräumliches Phänomen: Familien konzentrieren sich entweder in den Armutsvierteln der großen Städte oder - wenn sie es sich leisten können - in deren Umland, „im Grünen“. In den repräsentativen, die Öffentlichkeit prägenden

\footnotetext{
6 Zur Begründung wie auch zum Folgenden vgl. Kaufmann (2005, S. 215ff.). Zum selben Ergebnis kommt auf anderem Wege auch Birg (2001, S. 61).

7 Die Legitimationsformeln „Familienlastenausgleich“ und "Familienleistungsausgleich" werden in der politischen Diskussion unscharf verwendet. Beim Familien/astenausgleich geht es um distributive Entscheidungen, also um Bedarfsgesichtspunkte; beim Familien/eistungsausgleich um allo kative Entscheidungen, also um investive Gesichtspunkte (Werding 1998, S. 174ff.). Maßstab eines Familienleistungsausgleichs sollten die durch die Familienerziehung erzielten positiven externen Effekte für das Wirtschaftssystem sein.
} 
Gebieten der Großstädte kommen Kinder kaum mehr vor, hier dominieren die Kinderlosen. Nota bene bleiben rund die Hälfte aller Medienschaffenden ohne eigene Kinder. Familien und Kinder fehlen - von abstoßenden Skandalgeschichten und Produktwerbung abgesehen - deshalb auch in der medialen Öffentlichkeit weitgehend.

Eine direkte Konsequenz verbreiteter Kinderlosigkeit ist die Ausdünnung der Verwandtschaftsnetze, welche im Familiensektor nach wie vor eine erhebliche Ressource in Krisensituationen darstellen. Auch wenn Kinderlose in der Regel keineswegs als vereinsamt gelten müssen, scheint die Belastbarkeit ihrer privaten Netzwerke geringer. Es gibt bisher kaum Hinweise, dass die sogenannte Individualisierung, welche vorzugsweise den kinderarmen oder gar kinderfreien Sektor alternativer privater Lebensformen betrifft, funktionale Äquivalente für die traditionellen Solidaritäten hervorbringt.

Diese Problematik wird besonders deutlich in Fällen gesundheitlicher Beeinträchtigung, insbesondere in Fällen dauerhafter Hilfe- und Pflegebedürftigkeit. Schon heute ist offensichtlich, dass pflegebedürftige Menschen ohne engere Familienangehörige in höherem Maße in Heimen und ähnlichen öffentlichen Einrichtungen Unterkunft finden, was die öffentlichen Aufwendungen natürlich erhöht. Mit Bezug auf die Pflegeversicherung stellen Personen mit und ohne nahe Angehörige (Ehepartner, Kinder) Gruppen mit deutlich unterschiedlicher Risikostruktur dar. Die Gruppe ohne nahe Angehörige wird im Zuge der absehbaren allgemeinen Zunahme pflegebedürftiger Menschen überproportional wachsen.

Das dürfte nicht nur zu zusätzlichen Problemen für die ohnehin zunehmend belasteten öffentlichen Haushalte führen, sondern auch und vor allem zu personellen Problemen der Pflegekapazität. Schon heute wird der Engpass in den Pflegekapazitäten durch die Zivildienstleistenden überdeckt. Sollte der Zivildienst im Zuge einer Abschaffung der Wehrpflicht entfallen, und hierfür spricht nicht zuletzt die zunehmende Nachwuchsschwäche, so ist nicht erkennbar, woher sich Pflegepersonen in ausreichender Zahl rekrutieren lassen. Der fehlende Nachwuchs wird sich gerade in den sozialen Diensten bemerkbar machen, da hier arbeitssparenden Produktivitätssteigerungen besonders enge Grenzen ge- setzt sind. Deshalb wird sich auch der relative Preis der professionellen und semiprofessionellen Pflegeleistungen erhöhen. Auch der gelegentlich empfohlene Import ausländischer Pflegekräfte wird das Problem nicht lösen, zumal sie angesichts des allgemeinen Nachwuchsmangels bei entsprechender Qualifikation mit besser bezahlten Angeboten rechnen können. ${ }^{9}$ Eine einigermaßen menschenwürdige Lösung für die damit angedeuteten Probleme dürfte nur über eine Ausdehnung der nicht professionalisierten einheimischen Hilfeformen zu erreichen sein, seien sie entgeltlich oder unentgeltlich. Es liegt nahe, für die Rekrutierung vor allem an die Lebensphase der jungen Alten zu denken, für deren Beschäftigung unsere medialen Leitbilder eigentlich nur Konsum und Freizeit anbieten.

Die Annnahme scheint ziemlich unrealistisch, dass die gewerbliche Wirtschaft in größerem Umfang motiviert werden kann, ältere Arbeitslose einzustellen. Wahrscheinlich lässt sich die Bereitschaft erhöhen, ältere eingearbeitete und loyale Arbeitskräfte bis in ein höheres Alter zu beschäftigen. Auf dem allgemeinen Arbeitsmarkt bleibt jedoch der Konkurrenzvorteil der Jüngeren offensichtlich. Es wird also spezifischer Vorkehrungen und „moralischer Unternehmer" bedürfen, um erwerbslose Menschen in fortgeschrittenem Alter in produktive, in der Regel wohl wohnortnahe Tätigkeiten zu bringen. Und dies gilt wiederum vor allem für diejenigen, die keine Aufgaben in familialen Netzwerken finden können. Es ist eine skurrile, aber schon heute zu beobachtende Konstellation, dass immer mehr Großeltern um die Aufmerksamkeit von immer weniger Enkeln konkurrieren, von den kinderlosen Onkeln und Tanten ganz abgesehen. Die Frage wird dringlich, wie für Menschen, die mit oder ohne ihren Willen auf den regulären Arbeitsmärkten keine Chancen haben, Tätigkeitsfelder und Reziprozitätsverhältnisse erschlossen werden können. Denn die Legitimität eines bloßen „Ruhestandes“ wird angesichts der zu erwartenden steigenden Belastungen der erwerbstätigen Generationen und des verbesserten Gesundheitsund Bildungszustands der ,jungen Alten“ wohl zurückgehen.

\section{Schlussbemerkung}

Demografische Entwicklungen verlaufen langsam und unmerklich. Und sie haben kein Subjekt, auf das Einfluss genommen werden könnte. Die „Bevölkerung“ bleibt ein statistisches Konstrukt, auch wenn einige Kulturschaffende meinen, das ,deutsche Volk" an der Front des Reichstagsgebäudes durch „Bevölkerung“ ersetzen zu sollen. Wie auch immer man sie nennen mag: Nur insoweit kollektive Identität einem Gemeinwesen politische Handlungsfähigkeit verleiht, kann von Politik und erst recht von Sozialpolitik oder gesellschaftsgestaltender Politik die Rede sein. Die kollektive Identität der Bundesrepublik erscheint mir als in Deutschland akkulturiertem Schweizer weit stärker, als es die hiesige Öffentlichkeit wahrnehmen will. Der Globalisierungsdiskurs tut das Seine, um die eminent staatsgebundene Konstitution der europäischen Nationen infrage zu stellen. Und im Falle Deutschlands hat die Erinnerung an die rassistische Bevölkerungspolitik des Dritten Reichs die Beschäftigung mit Bevölkerungsfragen ebenso verhindert wie der Streit zwischen Konservativen und Liberalen bzw. Sozialdemokraten um das „richtige“ Familienverständnis und die damit verbundenen geschlechtsspezifischen Rollenbilder. Die Voraussetzungen sind deshalb in Deutschland denkbar ungünstig, um die Bevölkerungsfrage und insbesondere die Nachwuchsfrage zu einer zentralen Frage der kollektiven Zukunft zu machen, wie dies insbesondere in Frankreich, aber auch in Skandinavien gelungen ist.

\footnotetext{
Allerdings muss nicht damit gerechnet werden, dass die Pflegefälle proportional zur Menge der Hochaltrigen zunehmen. Denn die verlängerte Altersphase bezieht sich überwiegend auf das "Dritte Lebensalter", in dem gesundheitliche Beschwerden noch nicht den Alltag dominieren.

9 Einer Studie des Instituts für Christliche Sozialwissenschaften in Münster ( $H$. Geller) ist zu entnehmen: „Um Geld zu sparen, stellten viele ambulante Sozialdienste nicht oder nur unzureichend aus gebildetes Personal ein. Es komme häufig aus den ehemaligen Sowjetstaaten oder aus Polen. Bereits jetzt sei die Zahl der Auszubildenden an den hiesigen Pflegeschulen deshalb zurückgegangen" (Neue Westfälische, 10.9.2005). Überdies gibt es schon heute zu wenig praktische Ausbildungsplätze in diesem Bereich.
} 
Wie auch immer man hierzu stehen mag: Wer die Bevölkerungsfrage nicht als eine Frage der kollektiven Zukunft begreift, verabschiedet sich implizit von jeglicher Solidarität mit dem staatlich konstituierten Gemeinwesen, dem er angehört. In einer transnationalen Marktwirtschaft ist die Be- völkerungsfrage so lange kein Problem, wie sie sich in weitere Regionen der Welt ausdehnen und deren Humanressourcen als Produzenten und Konsumenten einbeziehen kann. Und das kann noch einige Jahrzehnte lang gut gehen. Aber gerade die älter werdenden Generationen orientieren sich an einem nationalen Horizont, und es ist keine europäische Lösung für die Fragen der Alterssicherung absehbar. Wer die Nachwuchsfrage nicht ernst nimmt, nimmt auch die Frage der alten Generationen nicht ernst.

\section{LITERATUR}

Berkel, B./Börsch-Supan, A./Ludwig, A./Winter, J. (2004): Sind Probleme der Bevölkerungsalterung durch eine höhere Geburtenrate lösbar?, in: Perspektiven der Wirtschaftspolitik 5/1, S. 71-90

Birg, H. (2001): Die demographische Zeitenwende - Der Bevölkerungsrückgang in Deutschland und Europa, München

Birg, H./Flöthmann, E. J./Frei, T./Ströker, K. (1998): Simulationsrechnungen zur Bevölkerungsentwicklung in den alten und neuen Bundesländern im 21. Jahrhundert. Materialien des Instituts für Bevölkerungsforschung und Sozialpolitik (IBS) der Universität Bielefeld, Bd. 45, Bielefeld

Borchert, J. (1993): Renten vor dem Absturz. Ist der Sozialstaat am Ende?, Frankfurt a. M.

Borchert, J. (2003): Der "Wiesbadener Entwurf“ einer familienpolitischen Strukturreform des Sozialstaats, in: Hessische Staatskanzlei (Hrsg.): Die Familienpolitik muss neue Wege gehen!, Wiesbaden

Dorbritz, J. (2005): Kinderlosigkeit in Deutschland und Europa - Daten, Trends und Einstellungen, in: Zeitschrift für Bevölkerungswissenschaft 30, S. 359-407

Ewerhart, G. (2003): Ausreichende Bildungsinvestitionen in Deutschland?, Beiträge zur Arbeitsmarkt- und Berufsforschung 266, Nürnberg Hansen, A. H. (1939): Economic Progress and Declining Population Growth, in: The American Economic Review, pp. 1-15

Henke, C. (2005): Zur Berechnung des Humankapitalbestands in Deutschland, iw-trends 1

Kaufmann, F.-X. (2005): Schrumpfende Gesellschaft - Vom Bevölkerungsrückgang und seinen Folgen, Frankfurt a. $M$.

Kaufmann, F.-X. (2007): Demografischer Wandel und gesellschaftliche Dynamik, in: Rehberg, K.-S. (Hrsg.): Die Natur der Gesellschaft. Verhandlungen des 33. Kongresses der Deutschen Gesellschaft für Soziologie, 9.-13. Oktober 2006 in Kassel, Frankfurt (im Erscheinen)
Keynes, J. M. (1937): Some Economic Consequences of a Declining Population, in: The Eugenics Review XXIX/1, pp. 13-17

Meyer, B. /Wolter, M. (2007): Demographische Entwicklung und wirtschaftlicher Strukturwandel - Auswirkungen auf die Qualifikationsstruktur am Arbeitsmarkt, in: Statistisches Bundesamt (Hrsg.): Neue Wege statistischer Berichterstattung - Mikro- und Makrodaten als Grundlage sozioökonomischer Modellierung (im Erscheinen)

Oeppen, J./Vaupel, J. W. (2002): Broken Limits to Life Expectancy,

in: Science 296, pp. 1029-1031

Romer, P. M. (1990): Endogenous Technological Change, in: Journal of Political Economy 98/5, Part 2, pp. 71-102

Sauvy, A. (1968): Population II: Population Theories, in: International Encyclopedia of the Social Sciences 12, pp. 349-357

Schirrmacher, F. (2004): Das Methusalem-Komplott, München

Schwarz, K. (1997): 100 Jahre Geburtenentwicklung, in: Zeitschrift für Bevölkerungswissenschaft 22, S. 481-491

Sinn, H. W. (2003): Ist Deutschland noch zu retten?, München Statistisches Bundesamt (2006): 11. koordinierte Bevölkerungs-Vorausberechnung: Annahmen und Ergebnisse, Wiesbaden

Werding, M. (1998): Zur Rekonstruktion des Generationenvertrags. Ökonomische Zusammenhänge zwischen Kindererziehung, sozialer Alterssicherung und Familienlastenausgleich, Tübingen

Wingen, M. (2003): Bevölkerungsbewusste Familienpolitik: Grundlagen, Möglichkeiten und Grenzen, Wien 